\title{
AVALIAÇÃO DE SINTOMAS DO PACIENTE COM CÂNCER DE BEXIGA EM CUIDADOS PALIATIVOS: ESTUDO DE CASO*
}

\author{
Bruna Eloise Lenhani ${ }^{1}$, Nen Nalu Alves das Mercês²
}

\begin{abstract}
RESUMO: O objetivo deste estudo foi avaliar os sintomas do paciente com câncer de bexiga em cuidados paliativos. Estudo de caso, realizado no período de março a junho de 2015, em um serviço hospitalar no sul do Brasil, com um participante do sexo masculino, com câncer de bexiga e a cuidadora principal (filha) de 47 anos. Realizada coleta de dados de prontuário físico, eletrônico e 12 avaliações de enfermagem, com a Escala de Avaliação de Sintomas Edmonton. Os sintomas predominantes foram: cansaço, sonolência, diminuição do apetite, depressão, ansiedade e bem-estar diminuído. O estudo sugere que os sintomas, quando não controlados, estão diretamente relacionados à menor qualidade de morte; observou-se a importância da utilização de uma escala específica para avaliação de sintomas de pacientes em cuidados paliativos, para melhorar a assistência prestada. DESCRITORES: Cuidados paliativos; Neoplasias da bexiga urinária; Enfermagem oncológica; Avaliação de sintomas.
\end{abstract}

\section{EVALUATION OF SYMPTOMS OF A PATIENT WITH BLADDER CANCER RECEIVING PALLIATIVE CARE: A CASE STUDY}

\begin{abstract}
This study aimed to assess the symptoms of a patient with bladder cancer receiving palliative care. It is a case study undertaken in March - June 2015, in a hospital service in the South of Brazil, with one male participant, with bladder cancer, and the main caregiver (daughter) who was 47 years old. Data was taken from the paper care records, electronic medical records, and 12 nursing assessments, using the Edmonton Symptom Assessment System (ESAS). The predominant symptoms were: tiredness, sleepiness, reduction in appetite, depression, anxiety, and reduced self-esteem. The study suggests that the symptoms - when not controlled - are directly related to lower quality of death; the importance was observed of using a specific scale for assessing the symptoms of patients receiving palliative care, in order to improve the care provided.
\end{abstract}

DESCRIPTORS: Palliative care; Urinary Bladder Neoplasms; Oncology nursing; Symptom assessment.

\section{EVALUACIÓN DE SÍNTOMAS DEL PACIENTE CON CÁNCER DE VEJIGA EN CUIDADOS PALIATIVOS: ESTUDIO DE CASO}

RESUMEN: La finalidad de este estudio fue evaluar los síntomas del paciente con cáncer de vejiga en cuidados paliativos. Estudio de caso, hecho en periodo de marzo a junio de 2015, en servicio de un hospital del sur de Brasil. Participaron un individuo del sexo masculino con cáncer de vejiga y la cuidadora principal (su hija) de 47 años. Los datos fueron obtenidos por medio de prontuario físico, electrónico y de 12 evaluaciones de enfermería, con la Escala de evaluación de Síntomas Edmonton. Los síntomas predominantes fueron: fatiga, somnolencia, disminución del apetito, depresión, ansiedad y bienestar reducido. El estudio sugiere que los síntomas, cuando no controlados, se asocian directamente a la menor calidad de muerte. Se observó la importancia de la utilización de una escala específica para evaluación de síntomas de pacientes en cuidados paliativos, con fines de mejorar la asistencia prestada.

DESCRIPTORES: Cuidados paliativos; Neoplasias de la vejiga urinaria; Enfermería oncológica; Evaluación de síntomas. *Artigo extraído da dissertação intitulada: “Qualidade de Morte em Cuidados Paliativos Oncológicos: estudos de casos
múltiplos". Universidade Federal do Paraná, 2015.

${ }^{1}$ Enfermeira. Mestre pela Universidade Federal do Paraná. Enfermeira do Hospital Erasto Gaertner. Curitiba, PR, Brasil. Enfermeira. Doutora em Enfermagem. Docente de Enfermagem da Universidade Federal do Paraná. Curitiba, PR, Brasil.

Autor Correspondente:

Recebido: $22 / 12 / 2016$

Bruna Eloise Lenhani

Finalizado: 15/09/2017

Universidade Federal do Paraná

R. Pref. Lothario Meissner, 632 - 80210-170 - Curitiba, PR, Brasil

E-mail: brulenhani@gmail.com 


\section{INTRODUÇÃO}

O câncer de bexiga é quatro vezes mais comum em homens do que em mulheres. Os fatores de risco que se destacam são o tabagismo e a exposição a produtos como tinta, borracha, couro, alumínio e arsênio ${ }^{(1)}$. O diagnóstico tardio se deve a sintomas pouco específicos, como disúria, hematúria e polaciúria. As chances de cura neste caso são diminuídas, portanto, há necessidade de ofertar cuidados paliativos (CP) que visam proporcionar qualidade de vida e morte digna ${ }^{(2)}$. A morte digna é considerada o processo natural de morrer, no qual os profissionais de saúde não visam acelerar e nem adiar, mas sim, proporcionar conforto e aliviar sintomas físicos e emocionais ${ }^{(3)}$.

Há vários instrumentos de avaliação de sintomas, dentre eles a Escala de Avaliação de Sintomas Edmonton (ESAS), desenvolvida na Universidade de Alberta em Edmonton, Canadá, com objetivo de avaliar sintomas comuns em pacientes com câncer e em cuidados paliativos, além de melhorar a gestão do atendimento ${ }^{(4)}$. Definiu-se a seguinte questão norteadora: Quais os sintomas apresentados pelo paciente com câncer de bexiga avançado? Como objetivo: avaliar os sintomas do paciente com câncer de bexiga em cuidados paliativos. Optou-se por estudar este caso, pois é incomum encontrar pacientes com câncer de bexiga em CP.

\section{- MÉTODO}

Trata-se de estudo de caso $^{(5)}$, sobre um paciente com câncer de bexiga em $\mathrm{CP}$, assistido em um hospital geral no sul do Brasil. O estudo de caso é definido como uma investigação empírica que busca em profundidade um fenômeno contemporâneo ${ }^{(5)}$ e possibilita a investigação desse fenômeno no contexto real da enfermagem, com diversas fontes de evidência que permitem a reflexão e a busca de alternativas para solução dos problemas ${ }^{(6)}$.

Após aprovação do projeto pelo Comitê de Ética em Pesquisa, com Parecer no: 970.798, o paciente e a cuidadora familiar foram convidadas a participarem do estudo assinando o Termo de Consentimento Livre e Esclarecido, segundo a Resolução 466/12(7).

A coleta de dados ocorreu semanalmente nos meses de março a junho de 2015. As fontes dos dados foram: prontuário físico, prontuário eletrônico e avaliação clínica. Os instrumentos utilizados: perfil sociodemográfico e clínico; Escala de Avaliação de Sintomas Edmonton (ESAS) e Performance Palliative Scale (PPS).

A PPS avalia as dimensões funcionais: capacidade de deambular, nível de atividade e evidência exterior de doença, autocuidado, ingesta oral e estado de consciência, com 11 níveis de performance e valores de 0 a $100 \%{ }^{(8)}$. A ESAS avalia nove sintomas: dor, cansaço, náusea, depressão, ansiedade, sonolência, apetite, falta de ar e bem estar, e o $10^{\circ}$ é de livre escolha, com escores de 0 a 10 . O paciente atribui uma nota de 0 a 10, sendo 0 a ausência do sintoma e 10 , a sua maior intensidade ${ }^{(4)}$.

A análise dos dados ocorreu através da estatística descritiva simples para os dados referentes ao PPS e ESAS. Utilizou-se o CARE Checklist ${ }^{(9)}$ na estruturação do estudo de caso.

\section{- RELATO DO CASO}

Identificação: 73 anos, sexo masculino, escolaridade ensino médio incompleto, casado, pai de nove filhos, sendo um filho falecido, aposentado por idade, trabalhou como mecânico industrial, natural do Rio de Janeiro-RJ, procedente de Araucária-PR, porém devido à doença e tratamento morava com uma filha em Curitiba, estado do Paraná.

História da doença atual: diagnosticado com carcinoma urotelial papilífero, de baixo grau invasor de lâmina própria em 2011 e submetido à cirurgia via transuretral (RTU). Não realizou seguimento do tratamento na instituição de saúde; no final de 2014, apresentou quadro álgico severo em região supra púbica e procurou atendimento no hospital cenário do estudo. Encaminhado ao serviço de $\mathrm{CP}$, realizou nova RTU, com segundo diagnóstico de carcinoma urotelial de alto grau, associado a metástase 
pulmonar e nódulo esplênico; no início de 2015 iniciou tratamento de quimioterapia paliativa; em junho de 2015 foi suspenso este tratamento, devido a insuficiência renal. Apresentou alterações degenerativas em coluna lombar; e em 26/06/2015 evoluiu a óbito no hospital.

O Quadro 1 apresenta o seguimento da avaliação dos sintomas da ESAS.

Quadro 1 - Avaliação dos sintomas do paciente com câncer de bexiga em cuidados paliativos. Curitiba, PR, Brasil, 2016

\begin{tabular}{|l|c|c|c|c|c|c|c|c|c|c|c|}
\hline \multirow{2}{*}{ Sem } & \multicolumn{10}{c|}{ Variáveis Avaliadas } \\
\cline { 2 - 13 } & Dor & Can & Son & Náu & Apet & Falta de ar & Dep & Ans & Bem estar & Outros & Total \\
\hline $1^{\mathrm{a}}$ & 3 & 9 & 7 & 5 & 7 & 0 & 5 & 7 & 5 & 5 & 530 \\
\hline $2^{\mathrm{a}}$ & 1 & 5 & 7 & 3 & 4 & 0 & 5 & 5 & 5 & 0 & 350 \\
\hline $3^{\mathrm{a}}$ & 8 & 8 & 8 & 5 & 7 & 3 & 5 & 5 & 6 & 0 & 550 \\
\hline $4^{\mathrm{a}}$ & 0 & 5 & 5 & 0 & 3 & 1 & 5 & 5 & 3 & 0 & 270 \\
\hline $5^{\mathrm{a}}$ & 0 & 4 & 4 & 0 & 4 & 1 & 7 & 7 & 7 & 3 & 370 \\
\hline $6^{\mathrm{a}}$ & 3 & 5 & 4 & 0 & 4 & 1 & 7 & 7 & 7 & 0 & 380 \\
\hline $7^{\mathrm{a}}$ & 3 & 5 & 4 & 0 & 4 & 1 & 7 & 7 & 7 & 0 & 380 \\
\hline $8^{\mathrm{a}}$ & 3 & 5 & 4 & 0 & 6 & 1 & 7 & 7 & 7 & 0 & 400 \\
\hline $9^{\mathrm{a}}$ & 5 & 5 & 4 & 2 & 7 & 2 & 7 & 7 & 7 & 0 & 460 \\
\hline $10^{\mathrm{a}}$ & 6 & 6 & 4 & 2 & 7 & 4 & 8 & 8 & 8 & 0 & 530 \\
\hline $11^{\mathrm{a}}$ & 6 & 7 & 5 & 2 & 7 & 4 & 8 & 8 & 8 & 0 & 550 \\
\hline $12^{\mathrm{a}}$ & 5 & 8 & 10 & 3 & 10 & 9 & & & & 7 & 3 \\
\hline
\end{tabular}

Legenda: Can - cansaço; Son - sonolência; Náu - náusea; Apet - apetite; Dep - depressão; Ans - Ansiedade

O Quadro 2 apresenta o resultado da avaliação do PPS.

Quadro 2 - Avaliação do PPS do paciente com câncer de bexiga em cuidados paliativos. Curitiba, PR, Brasil, 2016

\begin{tabular}{|c|c|c|c|c|c|c|c|c|c|c|c|c|}
\hline \multirow[t]{2}{*}{ PPS (\%) } & Avaliação & \multicolumn{11}{|c|}{ Avaliações de seguimento/Semanal } \\
\hline & $\mathbf{1}^{\mathrm{a}}$ & $2^{a}$ & $3^{a}$ & $4^{a}$ & $5^{a}$ & $6^{a}$ & $7^{a}$ & $8^{\mathbf{a}}$ & $9^{a}$ & $10^{\mathrm{a}}$ & $11^{\mathrm{a}}$ & $12^{\mathrm{a}}$ \\
\hline 1. Deambulação & \multirow[t]{5}{*}{70} & \multirow[t]{5}{*}{70} & \multirow[t]{5}{*}{60} & \multirow[t]{5}{*}{80} & \multirow[t]{5}{*}{70} & \multirow[t]{5}{*}{60} & \multirow[t]{5}{*}{60} & \multirow[t]{5}{*}{60} & \multirow[t]{5}{*}{50} & \multirow[t]{5}{*}{50} & \multirow[t]{5}{*}{50} & \multirow[t]{5}{*}{30} \\
\hline 2. Evidência da doença & & & & & & & & & & & & \\
\hline 3. Autocuidado & & & & & & & & & & & & \\
\hline 4. Ingesta Alimentar & & & & & & & & & & & & \\
\hline 5. Nível de Consciência & & & & & & & & & & & & \\
\hline
\end{tabular}

Foram 12 avaliações dos sintomas. As $1^{\mathrm{a}}, 3^{\mathrm{a}}, 4^{\mathrm{a}}, 6^{\mathrm{a}}, 7^{\mathrm{a}}$ e $9^{\mathrm{a}}$ realizadas no ambulatório, a $2^{\mathrm{a}}, 5^{\mathrm{a}}, 8^{\mathrm{a}}$ e $11^{\mathrm{a}}$ por tele monitoramento com o paciente, a $10^{\mathrm{a}}$ e $12^{\mathrm{a}}$ no internamento. Durante as avaliações, mantevese contactuante e participativo. Na avaliação do item "outros sintomas", referiu constipação, hematúria e na $11^{\text {a }}$ avaliação oligúria e edema de membros inferiores.

A $12^{\mathrm{a}}$ avaliação foi realizada com a cuidadora familiar principal - filha, de 47 anos, casada, natural e procedente de Curitiba-PR. O paciente estava dependente, acamado, com insuficiência renal, aceitação de dieta reduzida e oscilava com períodos de confusão mental e sonolência e PPS em $30 \%$. Os itens subjetivos não foram avaliados, conforme o guidelines da ESAS ${ }^{(4)}$. 
O carcinoma urotelial papilífero de alto grau caracteriza-se com taxa de proliferação rápida, probabilidade de recorrência um ano após a RTU e com probabilidade de progressão de 15 a $70 \%$, em cinco anos. Os principais sítios de metástase são fígado, pulmão e ossos ${ }^{(1)}$.

Um estudo randomizado mostrou que os pacientes submetidos a CP associados com terapia da dignidade (psicoterapia de curta duração para pacientes com doença limitante de vida) apresentaram efeito benéfico na intensidade de depressão e ansiedade, comparado aos que foram encaminhados somente aos $\mathrm{CP}^{(10)}$.

A fadiga acomete aproximadamente 50 a $90 \%$ dos pacientes com câncer, implicando na qualidade de vida de maneira severa ${ }^{(11)}$. O uso de metilfenidato e modanafil, agentes estimuladores de eritropoietina em casos de anemia e corticoides são utilizados na fase final de vida com fadiga grave; bem como terapias cognitivo-comportamentais (conservação de energia e organização de atividades diárias realizadas), exercícios físicos e terapias do sono ${ }^{(10)}$.

A anorexia pode levar a caquexia que compreende a astenia, hipoalbuminemia, emagrecimento e disfunção metabólica. Pode estar relacionada aos tratamentos anteriores, toxicidades tardias, menor expectativa de sobrevida e angústia psicossocial. O tratamento para anorexia inclui administração de metoclopramida e o uso de estimulantes do apetite, hormônio e corticosteroide ${ }^{(11)}$.

Estudo americano com o objetivo de identificar sintomas associados à síndrome do sono em paciente em CP concluiu que são mais propensos a relatar dor, depressão, ansiedade e diminuição no bem-estar $^{(12)}$.

O tratamento farmacológico para distúrbio do sono inclui as classes dos benzodiazepínicos e não-benzodiazepínicos hipnóticos, que possuem benefícios como manter latência do sono, redução do período de vigília, e melhoria da eficiência do sono ${ }^{(12)}$. O tratamento não farmacológico deve ser considerado como primeira linha para controle do distúrbio do sono e inclui a terapia comportamental cognitiva, controle de estímulos, redução do cochilo durante o dia, relaxamento e exercícios físicos ${ }^{(13)}$.

Outro aspecto importante em CP é a presença de um familiar; estudo brasileiro mostra que sua presença proporciona segurança e conforto, favorecendo a redução do tempo de internação; porém ressalta-se que este também precisam de apoio emocional, social e econômico ${ }^{(14)}$.

\section{CONSIDERAÇÕES FINAIS}

Dentre os sintomas que afetaram o paciente estudado foram: ansiedade, depressão, cansaço, sonolência e diminuição do apetite e bem-estar. Estes sintomas, quando não controlados estão relacionados, à menor qualidade de morte.

Para a enfermagem, destaca-se como ponto positivo a importância da ESAS para avaliação de sintomas em CP, pois fornece dados concretos sobre sua intensidade, favorecendo o planejamento para manejar os sintomas; bem como a necessidade de supervisionar o seguimento do paciente com câncer, evitando abandono do tratamento e consequente progressão da doença.

O método de estudo de caso apresenta limitações como a impossibilidade de generalizar os resultados obtidos, mas por outro lado contribui para o contexto em que o fenômeno foi investigado.

\section{REFERÊNCIAS}

1. Razmaria AA. Bladder Cancer. JAMA. [Internet] 2015;314(17) [acesso em 25 fev 2016]. Disponível: http://dx.doi. org/10.1001/jama.2015.13738.

2. do Prado BBF. Influência dos hábitos de vida no desenvolvimento do câncer. Cienc. Cult. [Internet] 2014;66(1) [acesso em 25 fev 2016] Disponível: http://dx.doi.org/10.21800/S0009-67252014000100011. 
3. World Health Organization (WHO). Cancer Control: Knowledge into action: WHO guide for effective programmes. [Internet] Geneva: WHO; 2007 [acesso em 24 fev 2016]. Disponível: http://www.who.int/cancer/ modules/Prevention\%20Module.pdf.

4. Alberta Health Services. Guidelines for Using the revised Edmonton Symptom Assessment System (ESAS- r). Alberta: Edmonton Zone Palliative Care Program; Alberta Health Services; 2009.

5. Yin RK. Estudo de caso: Planejamento e métodos. Porto Alegre: Bookman; 2015.

6. Gautério-Abreu DP, de Ávila JA, Santos SSC, Ilha S, da Silva BT. Contribuições do estudo de caso para o cuidado de enfermagem: um relato de experiência. Rev enferm UFPE on line. [Internet] 2016;10(3) [acesso em 01 jun 2017]. Disponível: https://periodicos.ufpe.br/revistas/revistaenfermagem/article/viewFile/11070/12501.

7. Ministério da Saúde (BR). Conselho Nacional de Saúde. Diretrizes e normas regulamentadoras de pesquisa envolvendo seres humanos. Resolução n. 466, de 12 de dezembro de 2012. Brasília; 2012

8. Tavares FAG. Acuidade prognóstica em fim de vida - valor preditivo de quatro métodos na estimativa de sobrevivência de doentes oncológicos de um hospital central e universitário português [dissertação]. Lisboa (POR): Faculdade de Medicina de Lisboa; 2010.

9. Case Reports. CARE Check List 2013. [Internet] 2013 [acesso em 20 nov 2016]. Disponível: http://www.carestatement.org/downloads/CAREchecklist-Portuguese.pdf.

10. Julião M, Barbosa A, Oliveira P, Nunes B, Carneiro AV. Efficacy of dignity therapy for depression and anxiety in terminally ill patients: Early results of a randomized controlled trial. Palliat Support Care. [Internet] 2013;11(6) [acesso em 14 fev 2016]. Disponivel: https://doi.org/10.1017/S1478951512000892.

11. National Comprehensive Cancer Network (NCCN). Palliative Care. Version 2. [Internet] NCCN; 2015 [acesso em 14 fev 2016]. Disponível: https://www.nccn.org/store/login/login.aspx?ReturnURL=https://www.nccn.org/ professionals/physician_gls/pdf/palliative.pdf.

12. Franceschini J, Jardim JR, Fernandes ALG, Jamnik S, Santoro IL. Relação entre a magnitude de sintomas e a qualidade de vida: análise de agrupamentos de pacientes com câncer de pulmão no Brasil. J. bras. pneumol. [Internet] 2013;39(1) [acesso em 13 fev 2016]. Disponível: http://dx.doi.org/10.1590/S1806-37132013000100004.

13. Gilbert JE, Howell D, King S, Sawka C, Hughes E, Angus H, et al. Quality improvement in cancer symptom assessment and control: the provincial palliative care integration project (PPCIP). J Pain Symptom Manage. [Internet] 2012;43(4) [acesso em 31 mai 2017]. Disponível: http://dx.doi.org/10.1016/j.jpainsymman.2011.04.028.

14. Paiva CE, Manfredini LL, Paiva BSR, Hui D, Bruera E. The Brazilian Version of the Edmonton Symptom Assessment System (ESAS) Is a Feasible, Valid and Reliable Instrument for the Measurement of Symptoms in Advanced Cancer Patients. PLoS. [Internet] 2015;10(7) [acesso em 24 nov 2015]. Disponível: http://dx.doi. org/10.1371/journal.pone.0132073. 